\title{
Steering Kernel Regression Total Variation for Image Denosing
}

\author{
Lin $\mathrm{Li}^{+}$, Xinhua Wei, Zhiyu Zuo and Wenjing Zhu \\ School of Agricultural Equipment Engineering, Jiangsu University, Zhenjiang 212013, China
}

\begin{abstract}
In this paper, a novel 11 minimization model was proposed for image denosing. We firstly proposed a new regularization term called Steering Kernel Regression Total Variation(SKRTV), which exploits the local structural regularity properties in natural images. By combining the SKRTV regularization term and global fidelity term, we proposed a maximum a posteriori probability framework of image denoising. Furthermore, split Bregman iteration was applied to implement the proposed model. Extensive experiments demonstrated the effectiveness of the proposed method.
\end{abstract}

Keywords: steering kernel regression, total variation, structural regularity, split Bregman iteration.

\section{Introduction}

Image denoising is an important and challenging inverse problem in image processing and computer vision since during the processes of being captured, digitized, recorded, and transmitted, an image is usually distorted and noisy. It refers to the recovery of a digital image that has been contaminated by some types of noise, while preserving image features such as the edges and the textures. Many approaches have been proposed to reconstruct the original image by exploiting the inherently spatial correlation. In these approaches, the use of variational methods and nonlinear partial differential equations (PDEs) [1] have significantly grown. The classical example is the total variation (TV) model, which was proposed by Rudin in [2] consisting of the minimization of the following energy that involves the total variation (TV) norm as an edge-preserving regularization functional:

The TV model has been proven to be quite effective for removing noise without causing excessive smoothing of the edges. However, it is well known that the TV model suffers from the so-called staircase effect, which may produce undesirable blocky images. With the development of machine learning techniques, non-parametric methods have been widely used in image processing problem, which rely on the data itself to dictate the structure of the model, in which case this implicit model is referred to as a regression function [3]. This family of methods is based on the assumption of local structural regularity, which says that there are meaningful structures in the spatial domain of natural images. Examples are bilateral filtering [4] and structure tensor based methods [5]. These methods utilize the local structural patterns to regularize the image processing procedure and based on the assumption that images are locally smooth except at edges.

In this paper, coupling the TV method and SKR method, we proposed a novel regularization term called Steering Kernel Regression Total Variation (SKRTV), which could both robustly obtain the local structure of images and effectively remove the noise. Split Bregman iteration was applied for the optimal solution due to the rapid convergence in dealing with TV-based optimization problem.

\section{The Proposed Methed}

\subsection{Steering kernel regression total variation}

\footnotetext{
Corresponding author. Tel.: +86 18651407687

E-mail address: lilin@ujs.edu.cn
} 
Steering kernel regression (SKR) is an effective tool for image reconstruction. The SKR framework defines its data model as:

$$
y_{i}=z\left(x_{i}\right)+\varepsilon_{i} \quad i=1, \ldots, P, x_{i}=\left[x_{1 i}, x_{2 i}\right]^{T}
$$

where $y_{i}$ is degraded sample at $x_{i}\left(x_{l i}\right.$ and $x_{2 i}$ are spatial coordinates $), z(\cdot)$ is the regression function to be estimated, $\varepsilon_{i}$ represents i.i.d. zero mean noise, $P$ stands for the total number of samples. The kernel regression framework provides a rich mechanism for computing point-wise estimates of the regression function with minimal assumptions about global signal or noise models. As a local method, the SKR uses $x$ near the sample $x_{i}$, and we have the Nth order Taylor series:

$$
z\left(x_{i}\right) \approx z(x)+\{\nabla z(x)\}^{T}\left(x_{i}-x\right)+\frac{1}{2}\left(x_{i}-x\right)^{T}\{H z(x)\}\left(x_{i}-x\right)+\cdots=\beta_{0}+\beta_{1}{ }^{T}\left(x_{i}+x\right)+\beta_{2}{ }^{T}\left\{\left(x_{i}-x\right)\left(x_{i}-x\right)^{T}\right\}+\cdots
$$

With:

$$
\beta_{1}=\nabla z(x)=\left[\frac{\partial z(x)}{\partial x_{1}}, \frac{\partial z(x)}{\partial x_{2}}\right]^{T} ; \quad \beta_{2}=\frac{1}{2}\left[\frac{\partial^{2} z(x)}{\partial x_{1}^{2}}, \frac{\partial^{2} z(x)}{\partial x_{1} \partial x_{2}}, \frac{\partial^{2} z(x)}{\partial x_{2}^{2}}\right]^{T}
$$

where $\nabla$ and $\mathrm{H}$ are the gradient $(2 * 1)$ and Hessian( $2 * 2)$ operators, respectively.

Since this method is based on local signal representations, a logical step is to estimate the parameters $\left(\beta_{n}\right)_{n=o}^{N}$ using all the neighboring samples $\left(y_{i}\right)_{i=1}^{p}$. A weighted least-square formulation of the fitting problem capturing this idea is:

$$
\min _{\left\{\beta_{n}\right\}_{n=0}^{N}} \sum_{i=1}^{P}\left[y_{i}-\beta_{0}-\beta_{1}^{T}\left\{\left(x_{i}-x\right)\left(x_{i}-x\right)^{T}\right\}-\cdots\right]^{2} * K H\left(x_{i}-x\right)
$$

With

$$
K H\left(x_{i}-x\right)=\frac{\sqrt{\operatorname{det}\left(C_{i}\right)}}{2 \pi h_{k}^{2}} \exp \left(-\frac{\left(x_{i}-x\right)^{T} C_{i}\left(x_{i}-x\right)}{2 h_{k}^{2}}\right)
$$

where $h_{k}$ is global parameter, $C_{i}$ is estimated as the local covariance matrix of the neighborhood spatial gradient vectors.

Regardless of the regression order and dimensionality of the regression function, we can reform it as a weighted least-square optimization problem:

$$
\hat{b}=\arg \min _{b}(y-X b)^{T} K(y-X b)
$$

where

$$
\begin{array}{rl}
y=\left[y_{1}, y_{2}, \ldots \ldots y_{p}\right]^{T}, \mathrm{~b} & =\left[\beta_{0}, \beta_{1}^{T}, \ldots \beta_{N}^{T}\right] \\
X & K=\operatorname{diag}\left[K_{H}\left(x_{1}-x\right), K_{H}\left(x_{2}-x\right), \ldots, K_{H}\left(x_{P}-x\right)\right] \\
& =\left[\begin{array}{cccc}
1 & \left(x_{1}-x\right)^{T} & \operatorname{vech}^{T}\left\{\left(x_{1}-x\right)\left(x_{1}-x\right)^{T}\right\} & \ldots \\
1 & \left(x_{2}-x\right)^{T} & \operatorname{vech}^{T}\left\{\left(x_{2}-x\right)\left(x_{2}-x\right)^{T}\right\} & \ldots \\
\vdots & \vdots & \vdots & \vdots \\
1 & \left(x_{n}-x\right)^{T} & \operatorname{vech}^{T}\left\{\left(x_{n}-x\right)\left(x_{1}-x\right)^{T}\right\} & \cdots
\end{array}\right]
\end{array}
$$

The solution to Eq.(5) is:

$$
\hat{Z}\left(x_{i}\right)=\hat{\beta}_{0}=e_{1}^{T}\left(X^{T} K X\right)^{-1} X^{T} K y=\sum_{i=1}^{P} \omega_{i}\left(K, H, N, x_{i}-x\right) y_{i}
$$

$e_{1}$ is a column vector, $\sum \omega_{i}=1$, we call $\omega_{i}$ the equivalent kernel weight for $y_{i}$.

Due to the ability of preserving edges, we extend steering kernel regression to bounded variation domain and propose a new regularization term called steering kernel regression total variation.

Given an observed image, the SKR gradient of an image at point $i$ is defined as:

$$
\nabla_{s} x(i, j):=(x(j)-x(i)) \sqrt{\omega(i, j)}
$$

where $\omega(i, j)$ is the SKR weight between $x_{i}$ and its similar pixel $x_{j}$, which has been defined in Eq.(4).

\subsection{The Proposed Model}


In order to exploit the image local correlation, we propose to use a novel regularization term based on steering kernel regression total variation (SKRTV) to learn a local structure prior. Along with the processing of the data to build the weight in Eq.(7), we expand the SKR to SKRTV, which provides an effective mechanism for the inverse problem.

$$
\left|\Delta_{S} X\right|_{1}=\sum_{i \in X_{i}, j \in P\left(X_{i}\right)}\left|(X(j)-X(i)) \sqrt{\omega_{s}(i, j)}\right|_{1}
$$

where $P\left(X_{i}\right)$ denotes the neighbors of $X_{i} . \omega_{s}(i, j)$ is the SKR weight between $X_{i}$ and its similar pixel $X_{j}$.

The SKRTV term makes the local smoothness assumption that a target pixel can be weighted average from its neighbors as the SKR term, which can exploit the local structural regularity in natural images. By expanding SKR to the SKRTV, we can obtain more satisfactory results with sharper edges. We can estimate $X$ by solving:

$$
\hat{X}=\min _{X}\left|\Delta_{S} X\right|_{1}+\frac{\lambda}{2}\|X-Y\|_{2}^{2}
$$

where $\lambda$ is a trade-off parameter. Steering Kernel Regression can robustly obtain the local structure of images by analyzing the pixel value differences based on estimated gradients. In the SKR, the weights or kernels locally adapt to the underlying structure of the image. The weights follow locally dominant directions and dictate the contribution of various pixels in a local neighborhood of the pixel to be denoised. By expanding SKR to the SKRTV, we can obtain images with sharper edges.

\subsection{Implement of The Proposed Model}

In order to effectively solve the proposed model, we adopt the split Bregman iteration method due to the rapid convergence in dealing with TV-based optimization problem. The key to the split Bregman iteration is "de-couple" the 11 and 12 portions of the energy in Eq.(9). The idea is to reformulate the problem as:

$$
\min _{X, d}|d|_{1}+\lambda H(x) \text {, subject to } d=\nabla_{S} X, H(X)=\frac{1}{2}\|X-Y\|_{2}^{2}
$$

To solve this problem, it is firstly converted into an unconstrained problem:

$$
\min _{X, d}|d|_{1}+\lambda H(X)+\frac{\mu}{2}\left\|d-\nabla_{s} X\right\|_{2}^{2}
$$

The above problem can be modified as the following iterative procedure:

$$
\left(X^{k+1}, d^{k+1}\right)=\min _{X, d}|d|_{1}+\lambda H(X)+\frac{\mu}{2}\left\|d-\nabla_{S} X\right\|_{2}^{2}, \quad b^{k+1}=b^{k}+\left(\nabla_{S}\left(X^{k+1}\right)-d^{k+1}\right)
$$

The solution to Eq.(13) can be performed efficiently by iteratively minimizing with respect to $X$ and $d$ separately. The two steps we must perform are:

$$
\begin{aligned}
& \text { step } 1: X^{k+1}=\arg \min _{X}\left(\lambda H(x)+\frac{\mu}{2}\left\|d-\nabla_{S} X-b^{k}\right\|_{2}^{2}\right) \\
& \text { step } 2: d^{k+1}=\arg \min _{d}\left(\lambda|d|_{1}+\frac{\mu}{2}\left\|d-\nabla_{s} X-b^{k}\right\|_{2}^{2}\right)
\end{aligned}
$$

To solve step1, the optimization problem we must solve for $X^{k}$ is now differentiable, we can thus use a wide variety of optimization techniques to solve this problem. In step2 of the above algorithm, there is no coupling between elements of $d$, we can explicitly compute the optimal value of $d$ by shrinkage operators.

\section{Acknowledgements}

To validate the effectiveness of the proposed method, we conduct experiments on various images. Several state-of-the-art methods, such as BM3d [6], NLTV [1], SKR [3], KSVD [7] and PLOW[8], are used as comparison baselines. The peak signal-to-noise (PSNR) and the structural similarity (SSIM) are employed to illustrate the quantitative quality of the denoised results. Since the human visual system is more sensitive to the luminance channel than the chrominance channels, we only use grayscale images in this paper.

The denoising results with different methods on two grayscale images: Lena and Monarch are shown in Table 1, where the bold parts imply the best effects. From Table 1, we can observe that the original SKR method has lower PSNR and SSIM values because 12-based regularization may blur edges during denosing 
process. Our method significantly outperforms the NLTV, KSVD and PLOW in the most part. To further illustrate the effectiveness of the proposed method, the results of grayscale image "Lena" for perceptual quality comparison are shown in Figure 1. We can observe that the non-local TV method is able to effectively suppressing noise, but it generates obvious jaggy artifacts along edges. The SKR method could keep better structure, but it blurs edges. Although the learning-based method (KSVD) can produce some high-frequency details, the resulted images appear to be unnatural. PLOW tends to oversmoothing edges.

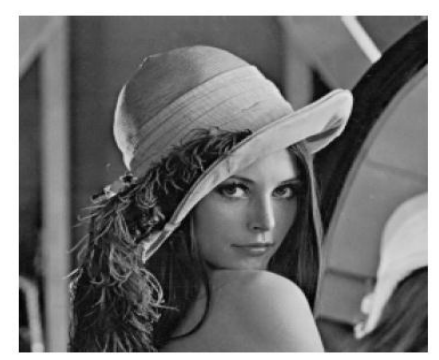

(a) Original image

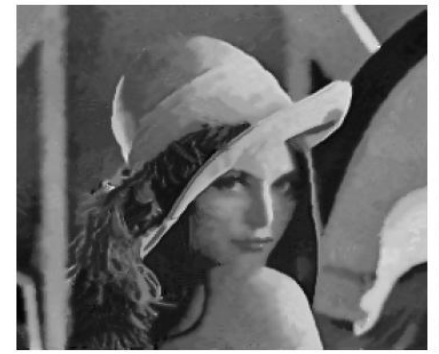

(e) NLTV

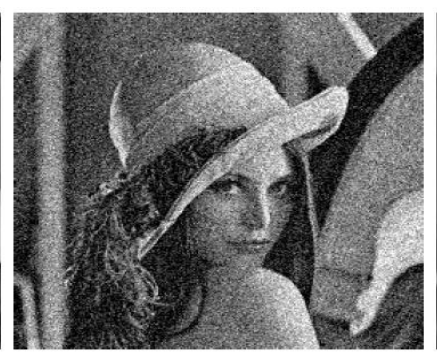

(b) Noisy image

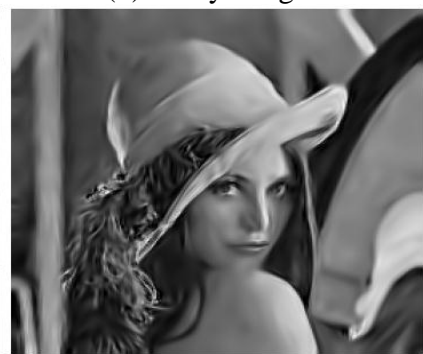

(f) SKR

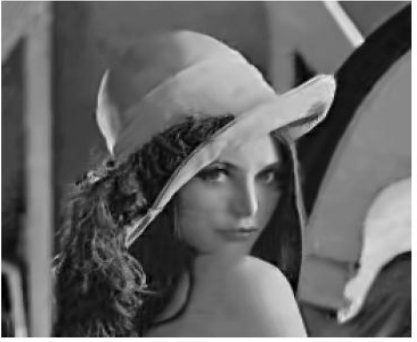

(c) The proposed method

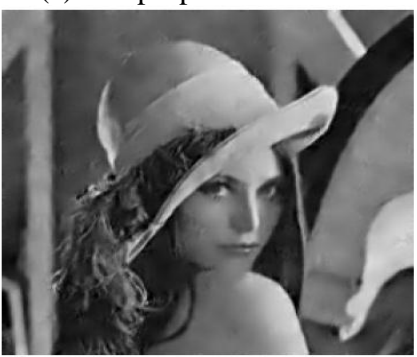

(g) KSVD

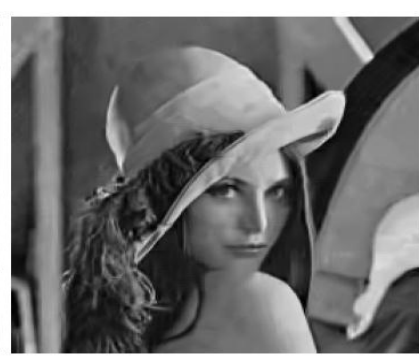

(d) BM3D

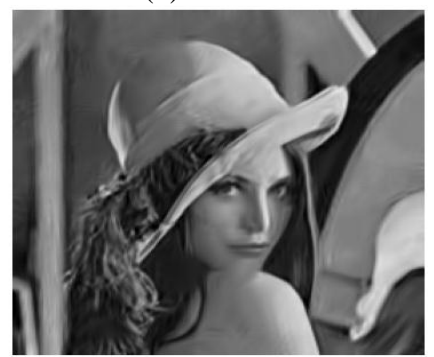

(h) PLOW

Fig. 1: Comparison of visual results by different methods on "Lena" with $\sigma=30$.

Table 1: Performance of Different Methods: PSNR and SSIM Results on Two Images

\begin{tabular}{|c|c|c|c|c|c|c|c|c|c|c|c|c|c|}
\hline \multirow{2}{*}{\multicolumn{2}{|c|}{ Images }} & \multicolumn{6}{|c|}{ Lena } & \multicolumn{6}{|c|}{ Monarch } \\
\hline & & SKRTV & BM3D & NLTV & SKR & KSVD & PLOW & SKRTV & BM3D & NLTV & SKR & KSVD & PLOW \\
\hline \multirow{2}{*}{$\sigma=10$} & PSNR & 34.49 & 34.48 & 33.33 & 33.69 & 33.98 & 33.33 & 35.00 & 34.56 & 33.79 & 33.06 & 34.01 & 34.07 \\
\hline & SSIM & 0.931 & 0.931 & 0.910 & 0.914 & 0.926 & 0.919 & 0.971 & 0.969 & 0.947 & 0.946 & 0.961 & 0.962 \\
\hline \multirow{2}{*}{$\sigma=20$} & PSNR & 30.94 & 30.98 & 29.91 & 30.37 & 30.40 & 30.07 & 30.93 & 30.66 & 30.09 & 29.13 & 30.19 & 29.71 \\
\hline & SSIM & 0.883 & 0.882 & 0.854 & 0.872 & 0.861 & 0.871 & 0.937 & 0.935 & 0.910 & 0.917 & 0.922 & 0.926 \\
\hline \multirow{2}{*}{$\sigma=30$} & $\begin{array}{l}\text { PSNR } \\
\end{array}$ & 28.89 & 28.90 & 28.05 & 28.26 & 28.37 & 28.49 & 28.69 & 28.64 & 27.93 & 27.11 & 28.00 & 27.90 \\
\hline & SSIM & 0.844 & 0.839 & 0.808 & 0.833 & 0.818 & 0.832 & 0.902 & 0.901 & 0.850 & 0.878 & 0.882 & 0.891 \\
\hline
\end{tabular}

\section{References}

[1] X. Zhang, M. Burger, et ac, "Bregmanized Nonlocal Regularization for Deconvolution and Sparse Reconstruction, ” SIAM J. Imaging Sciences, 253-276, (2010).

[2] Leonid.I.Rudin, S.Osher, E.Fatemi, "Nonlinear total variation based noise removal algorithms, " Physica D: Nonlinear Phenomena, 259-268, (1992).

[3] H. Takeda, S. Farsiu, and P. Milanfar, "Kernel Regression for Image Processing and Reconstruction," IEEE Transactions on Image Processing, 349-366, (2007).

[4] H. Zhang, J. Yang, Y. Zhang, and T. S. Huang, "Non-local kernel regression for image and video restoration, "In $\operatorname{ECCV(3):~566-579,~(2010).~}$

[5] H. Takeda, S. Farsiu, P. Milanfar, "Robust Kernel Regression for Restoration and Reconstruction of Images from Sparse Noisy Data, ” In ICIP, 1257-1260, (2006).

[6] K. Dabov, A. Foi, V. Katkovnik,et ac, "Image Denoising by Sparse 3-D Transform Domain Collaborative Filtering, " IEEE Trans. on Image Processing, 2080-2095, (2007).

[7] M. Elad, M. Aharon, "Image Denoising via Sparse and Redundant Representations over Learned Dictionaries," IEEE Trans. on Image Processing, 3736-3745, (2006).

[8] P.Chatterjee, P.Milanfar, Patch-Based Near-Optimal Image Denoising, IEEE Trans. on Image Processing,16351649, (2012). 